\title{
Analysis of a Hybrid Multicode/Variable Spreading Factor DS-CDMA System With Two-Stage Group Detection
}

\author{
Dong In Kim, Senior Member, IEEE
}

\begin{abstract}
High-rate uplink transmission is realized by proposing a hybrid multicode (MC)/variable spreading factor (VSF) direct-sequence code-division multiple-access system that employs precoding for a constant envelope MC signal. In connection with the hybrid MC/VSF and precoding scheme, a novel two-stage $M$-symbol group detection is introduced to maximize the detection gain by collecting all the signal energy carried over the entire $M$ MC channels. With fixed $M$, the symbol-error rate of the proposed hybrid scheme is derived for varying spreading factor per symbol, considering both identically and nonidentically distributed channel statistics. While the information rate and average transmit power per bit are kept the same, it is shown that, with manageable receiver complexity, the hybrid scheme can be a good alternative to multidimensional MC or, even better, outperforming classical MC and VSF in view of the bit-error rate.
\end{abstract}

Index Terms-Constant envelope, direct-sequence code-division multiple access (DS-CDMA), high-rate uplink transmission, hybrid multicode/variable spreading factor (MC/VSF), precoding, two-stage group detection.

\section{INTRODUCTION}

$\mathbf{T}$ HE NECESSITY of high-rate transmission over wireless channels is rapidly growing due to an increasing demand of data services. However, typical approaches to providing the data rates above $2 \mathrm{Mb} / \mathrm{s}$ have mostly been devised for the downlink due to traffic asymmetry and power limitation [1]. Especially in a cellular environment, the uplink channel is power limited due to a small size handset and is also affected by a nonlinear distortion since a cost-effective and power-efficient amplifier is typically required. With the facts, it is very hard to increase the rate up to $2 \mathrm{Mb} / \mathrm{s}$ in the uplink while meeting the quality-of-service (QoS) requirements. Besides, the multilevel modulation to increase the rate is not suitable for high-rate uplink transmission because of the nonlinear distortion.

For this reason, direct-sequence code-division multiple access (DS-CDMA) is well combined with the variable spreading factor (VSF) scheme to offer variable and high data rates [2], [3]. By adapting the spreading factor (SF) to the data rates with fixed chip rate, it avoids the nonlinear distortion and also results in simplified receiver structure. However, a maximum achievable data rate is actually limited by any minimum value of SF

Manuscript received June 10, 2003; revised October 6, 2003 and December 22, 2003. This work was supported by Simon Fraser University under a PRG Research Grant.

The author is with the School of Engineering Science, Simon Fraser University, Burnaby, BC V5A 1S6, Canada (e-mail: dikim@sfu.ca).

Digital Object Identifier 10.1109/TVT.2004.825786 to be required in order to meet a given QoS. Meanwhile, to circumvent this limitation, we may consider the multicode (MC) option for DS-CDMA, which inserts more parallel channels in proportion to the data rates and maintains a constant SF [4], [5]. However, it requires precoding to achieve a constant envelope by which some redundancy is added [6]-[8]. When detection is performed per parallel channel, the signal energy carried over precoding channels is not collected so that the BER is degraded. Such degradation in BER becomes severe when a maximum data rate is serviced and limits the maximum number of MC channels, i.e., the maximum data rate.

Based on the above observations, a hybrid MC/VSF scheme is proposed to meet the uplink channel requirements through a compromise between the minimum SF and the maximum number of MC channels. To overcome the BER degradation by precoding, a two-stage $M$-symbol group detection is introduced to collect all the signal energy embedded in the entire $M \mathrm{MC}$ channels. Compared to the MC option, the hybrid scheme requires much less $\mathrm{MC}$ channels so that the symbol detection can be made with a modest increase in receiver complexity. In particular, the multidimensional MC (MDMC) DS-CDMA scheme has been proposed in [9] to further increase the data rate by compensating for the loss in information rate by precoding. A symbol-by-symbol detection was first used to mitigate the bit-error rate (BER) degradation by precoding, but restricted to $J$-parallel symbol detection with $J \ll M$. Even with $M$-parallel symbol detection, the complexity order here is kept the same as $J$-parallel symbol detection of the MDMC scheme with the aid of the two-stage group detection.

With fixed $M$, the hybrid MC/VSF scheme is analyzed in terms of the symbol-error rate (SER) for varying SF to provide variable and high data rates, where both identically and nonidentically distributed channel statistics are considered. In addition, two-antenna diversity is employed at the receiver to offer a reliable uplink even with high data rates. Then, the hybrid scheme is compared with the MDMC in terms of the BER while the data rate is kept constant with comparable receiver complexity. To show superiority over classical MC and VSF, the BER of the hybrid scheme is also compared with the corresponding BERs, even though there is a modest increase in receiver complexity due to the $M$-parallel symbol detection.

The rest of the paper is organized as follows. In Section II, the system model is given, in which a high-rate user coexists with interfering low-rate users and the hybrid MC/VSF signaling is modeled for uplink high-rate transmission. To realize the $M$-symbol detection, we propose a two-stage group detection 
in Section III for which decision statistics are formulated. Section IV derives approximate bounds on the SER performance for the two-stage group detection, which result in tractable definite integral forms. Theoretical and simulation results are presented in Section V to verify the improvement in SER by the group detection. Finally, concluding remarks are given in Section VI.

\section{SYSTEM MODEL}

The hybrid MC/VSF scheme can be viewed as a special form of the MC option, while the SF can be reduced to provide the data rates above a certain limit. Assume that there are $M$-parallel MC channels in the MC option given the SF is $N$ chips per symbol. Then, the hybrid scheme adjusts the SF to be $N_{i}=$ $N / 2^{i}, i=1,2, \ldots$, as the data rate increases, under the constraint $N_{i} \geq M$.

A general format of MC signaling can be found in [10] and [11], where there exists only the difference between them in the way that concatenated channelization (orthogonal) codes are formed. This paper is concerned with the comparison between $\mathrm{MC} / \mathrm{VSF}$ and MDMC, including VSF and MC and, hence, it is necessary to adopt the same MC signal format as proposed in [9] while MD signaling is replaced by the VSF option here to adjust the SF for higher data rates.

Following the MC format as given in [9], the hybrid MC/VSF baseband signal can be expressed by

$$
\begin{aligned}
s(t)= & \sum_{n=0}^{N_{f}-1} \sum_{g=0}^{G_{i}-1} \sum_{m=0}^{M-1} \sqrt{P_{i}} \\
& \cdot\left[d_{I, m}(n) c_{I}\left(t-g T_{w}-n T_{i}\right)\right. \\
& \left.\quad+j d_{Q, m}(n) c_{Q}\left(t-g T_{w}-n T_{i}\right)\right] \\
& \cdot w_{m}\left(t-g T_{w}-n T_{i}\right)
\end{aligned}
$$

where $P_{i}$ is the signal power per channel, $\left\{d_{O, m}(n), m=\right.$ $0,1, \ldots, M-1\}(O=I, Q)$ is the $M$-parallel binary data at the $n$th signaling time, $T_{w}$ and $T_{i}=G_{i} T_{w}$ denote the Walsh and data symbol times, respectively, $M G_{i}=N_{i}$ is the SF (variable), and $N_{f}$ is the frame length.

The Walsh code waveform $w_{m}(t), m=0,1, \ldots, M-1$, is given by

$$
w_{m}(t)=\sum_{l=0}^{M-1} w_{m, l} p\left(t-l T_{c}\right)
$$

where $p(t)$ is a rectangular chip pulse occupied in $\left[0, T_{c}\right)$ with unit magnitude and $\left(w_{m, 0}, \ldots, w_{m, M-1}\right)$ denotes the $m$ th row vector of Hadamard matrix $H_{M}(M \times M)$ with elements \pm 1 . In order to have an in-phase $(I)$ /quadrature $(Q)$ code-multiplexed channel, the spreading code waveform is designed as

$$
\begin{aligned}
& c_{O}\left(t-g T_{w}-n T_{i}\right) \\
& \quad=\sum_{l=0}^{M-1} c_{O, n N_{i}+g M+l} p\left(t-\left(n N_{i}+g M+l\right) T_{c}\right)
\end{aligned}
$$

where the (fixed) chip time is given by $T_{c}=T_{i} / N_{i}=T_{w} / M$.

To illustrate the MC/VSF signaling, for instance, we assume that $M=16, N=128$, and $G=N / M=8$. When a precoding of rate 9/16 is employed, 9-b information can be sent on 16 parallel MC channels during the symbol time $T\left(=2^{i} T_{i}\right)=128 T_{c}$.
To increase the rate to $18 \mathrm{~b}$ per symbol, the hybrid MC/VSF reduces the SF to $N_{1}=N / 2=64$ and $G_{1}=G / 2=4(i=1)$ with (fixed) $M=16$. However, if the MC option is employed, it is required to increase $M \geq 32$ for a constant envelope. Likewise, for $36 \mathrm{~b}$ per symbol, the hybrid scheme reduces the SF to $N_{2}=N / 4=32$ while the MC option should increase $M \geq 64$, which is not feasible for practical implementation. Furthermore, the hybrid MC/VSF can increase the data rate up to $72 \mathrm{~b}$ per symbol (128 chips) using $M=16$-parallel MC channels where the $\mathrm{SF}$ is reduced to $N_{3}=N / 8=16$, while satisfying the constraint $N_{i} \geq M$. Note that this is equivalent to the effective SF per bit less than 2 in case of the VSF.

For comparison, the MDMC signaling is introduced as proposed in [9]

$$
\begin{aligned}
s(t)= & \sum_{n=0}^{N_{f}-1} \sum_{g=0}^{G-1} \sum_{m=0}^{M-1} \sqrt{P} \\
& \cdot\left[d_{I, m}(n) b_{I,\langle m\rangle}(g ; n) c_{I}\left(t-g T_{w}-n T\right)\right. \\
& \left.\quad+j d_{Q, m}(n) b_{Q,\langle m\rangle}(g ; n) c_{Q}\left(t-g T_{w}-n T\right)\right] \\
& \cdot w_{m}\left(t-g T_{w}-n T\right)
\end{aligned}
$$

where the $G$-ary orthogonal signaling by the modulating sequence $\left\{b_{O,\langle m\rangle}(g ; n), g=0,1, \ldots, G-1\right\}(O=I, Q)$ is used on each subset of $J(\leq M)$ MC channels (for details, refer to [9]). Here, $\langle m\rangle \triangleq\lfloor m / J\rfloor$ takes an integer from $\{0,1, \ldots, Q-$ $1\}$ with $Q=M / J$, where $\lfloor x\rfloor$ is the greatest integer not exceeding $x$. For instance, if $M=16, J=4, N=128$, and $G=8$ are assumed, the MDMC can send $18 \mathrm{~b}$ per symbol using the precoding of rate $9 / 16$ as before. In this case, the MDMC can be compared with the hybrid MC/VSF having the SF $N_{1}=64$ at the same data rate.

A high-rate user will choose either the hybrid MC/VSF signal in (1) or the MDMC signal in (4), while $(K-1)$ interfering low-rate users can be simply modeled as

$s_{k}(t)=\sum_{l=0}^{N N_{f}-1} \sqrt{\Gamma_{k}}\left[a_{I, l}^{(k)}+j a_{Q, l}^{(k)}\right] p\left(t-l T_{c}\right), \quad k=2, \ldots, K$

where $\Gamma_{k}$ is the $k$ th low-rate user's signal power and $\left\{a_{O, l}^{(k)}, l=0,1, \ldots,\right\}$ are assumed to be random binary sequences. Note that the bit energy is set to $E_{b}=\Gamma_{k} N_{k} T_{c}$ and $N_{k}$ will determine the bit rate of the $k$ th low-rate user.

Generally, a high-rate user is more affected by the self-interference due to a multipath fading in wireless channels and two receive antennas are used in order to provide space diversity where $V$ resolvable multipaths are modeled as

$$
h_{k}^{(q)}(t)=\sum_{v=1}^{V} \beta_{k, v+q V} \delta\left(t-\tau_{k, v}\right)
$$

for the $q$ th $(q=0,1)$ antenna of the $k$ th user link. It is assumed that $\left\{\tau_{k, v}\right\}$ are uniformly distributed over $[0, T)$ and that $\left\{\beta_{k, v+q V}, k=1, \ldots, K ; v=1, \ldots, V ; q=0,1\right\}$ is a set of complex-valued Gaussian random variables. Then, the received signal at the $q$ th antenna can be expressed by

$$
r_{q}(t)=s(t) \otimes h_{1}^{(q)}(t)+\sum_{k=2}^{K}\left[s_{k}(t) \otimes h_{k}^{(q)}(t)\right]
$$

for $\otimes$, denoting the convolution operation. 


\section{Two-Stage GRoup Detection}

For a high-rate user using the MC/VSF signal in (1), the twostage group detection is realized in connection with precoding, in order to increase the detection gain without causing much complexity in the receiver. First, we assume $M=16$ parallel MC channels for which the precoding of rate $9 / 16$ is designed to yield a constant envelope MC signal. The precoding designed here can be viewed as a nonlinear block coding technique by which some redundancy is added. The rule for adding redundancy, called the precoding rule, is determined such that the constant envelope property is maintained after superposition of MC channels.

Let us define

$$
\alpha^{l} \triangleq\left[d_{O, 4 l}(n), d_{O, 4 l+1}(n), d_{O, 4 l+2}(n), d_{O, 4 l+3}(n)\right]
$$

for $O=I, Q ; l=0,1,2,3$, where $\left[\alpha^{0}\left|\alpha^{1}\right| \alpha^{2} \mid \alpha^{3}\right]$ represents the $M=16$ parallel binary data $\left\{d_{O, m}(n)\right\}$. Then, the design procedure for the precoding is described as follows.

1) For each of the first three symbols (12 b) $\left[\alpha^{0}\left|\alpha^{1}\right| \alpha^{2}\right], 1-b$ precoding at the first level is performed to yield four-tuple vectors with odd weights of ones, respectively, where the first-level precoding rule is defined as

$$
d_{O, 4 l+3}(n)=-d_{O, 4 l}(n) \cdot d_{O, 4 l+1}(n) \cdot d_{O, 4 l+2}(n)
$$

for each 3-b information $\left\{d_{O, 4 l+j}(n)\right\}(j=0,1,2)$, $l=0,1,2$.

2) The second-level precoding rule is defined such that the last precoding symbol (4 b) $\alpha^{3}$ can be determined as

$$
\begin{aligned}
\alpha^{3}=-\frac{1}{2}\left(\left[\frac{1}{2}\left(\alpha^{0} H_{4}\right)\right] \odot\left[\frac{1}{2}\left(\alpha^{1} H_{4}\right)\right]\right. \\
\left.\odot\left[\frac{1}{2}\left(\alpha^{2} H_{4}\right)\right]\right) H_{4}
\end{aligned}
$$

where $H_{4}=\left[(1,1,1,1)^{T}\left|(1,-1,1,-1)^{T}\right|\right.$ $\left.(1,1,-1,-1)^{T} \mid(1,-1,-1,1)^{T}\right]$ for the transpose operation $T$ and $\odot$ denotes the vector product.

Note that the above mapping is equivalent to the precoding of rate $9 / 16$, where details are found in [7]. Hence, the precoding inserts 7-b redundancy to carry 9-b information on $M=16$ parallel MC channels.

Here, the symbol $\alpha^{l}$ can choose any one of $\alpha_{0}=$ $(-1,-1,-1,1), \quad \alpha_{1}=(-1,-1,1,-1), \quad \alpha_{2}=$ $(-1,1,-1,-1), \alpha_{3}=(-1,1,1,1)$, and $\alpha_{e}=-\alpha_{e-4}$ for $e=4,5,6,7$. Thus, the set of symbols $\left\{\alpha_{e} \mid e=0, \ldots, 7\right\}$ becomes biorthogonal signals, which allow us to realize the two-stage group detection as follows. Now, a group symbol is selected to be $\left[\alpha^{0}\left|\alpha^{1}\right| \alpha^{2} \mid \alpha^{3}\right]$ subject to the constraint $\alpha^{l}=\alpha_{e}$, $e=0,1,2,3$ for $l=0,1,2$ while $\alpha^{3}$ is precoded. Let us define $A_{j} \triangleq\left[\alpha^{0}\left|\alpha^{1}\right| \alpha^{2} \mid \alpha^{3}\right]$, where the cardinality $\left|\left\{A_{j}\right\}\right|$ equals $4^{3}=64 .{ }^{1}$ Then, there are 64 group symbols, while each group consists of eight distinct elements such as

$$
\begin{aligned}
& A_{j / 0}=\left[+\alpha^{0}\left|+\alpha^{1}\right|+\alpha^{2} \mid+\alpha^{3}\right] \\
& A_{j / 1}=\left[+\alpha^{0}\left|+\alpha^{1}\right|-\alpha^{2} \mid-\alpha^{3}\right] \\
& A_{j / 2}=\left[+\alpha^{0}\left|-\alpha^{1}\right|+\alpha^{2} \mid-\alpha^{3}\right] \\
& A_{j / 3}=\left[-\alpha^{0}\left|+\alpha^{1}\right|+\alpha^{2} \mid-\alpha^{3}\right]
\end{aligned}
$$

${ }^{1}$ If the two-level precoding designed here were not employed for $M=16$ parallel MC channels, the group detection should have realized $8^{4}=4096$-ary symbol detection, which seems to not be feasible. and $A_{j / e}=-A_{j / e-4}$ for $e=4,5,6,7$. Here, the sign of $\alpha^{3}$ can be determined by the precoding rule from (9). It is to be noted that all elements $\left\{A_{j / e}\right\}$ are equivalent to $A_{j}$ with respect to the group detection as follows.

In order to realize the group detection, we first form decision statistics, which is given by

$$
\begin{aligned}
X_{m, g}(n ; v+q V)= & \frac{1}{T_{w} \sqrt{P_{i}}} \int_{g T_{w}+n T_{i}+\tau_{1, v}}^{(g+1) T_{w}+n T_{i}+\tau_{1, v}} r_{q}(t) \\
& \cdot c_{O}\left(t-g T_{w}-n T_{i}-\tau_{1, v}\right) \\
& \cdot w_{m}\left(t-g T_{w}-n T_{i}-\tau_{1, v}\right) d t
\end{aligned}
$$

for $m=0, \ldots, M-1 ; g=0, \ldots, G_{i}-1 ; v=1, \ldots, V$; and $q=0,1$. If two-antenna diversity is applied to the $I$-channel statistics, then the maximal-ratio combining (MRC) with uncorrelated $2 \mathrm{~V}$ paths yields

$$
X_{\langle m\rangle}(n)=\sum_{v=1}^{2 V} \operatorname{Re}\left\{\beta_{1, v}^{*} X_{\langle m\rangle}(n ; v)\right\}
$$

where the matrix $X_{\langle m\rangle}(n ; v)$ has the elements $\left\{X_{m, g}(n ; v) \mid m=4\langle m\rangle, \ldots, 4\langle m\rangle+3 ; g=0, \ldots, G_{i}-1\right\}$. * denotes the complex conjugate. Note that for the $Q$-channel statistics, the real part $\operatorname{Re}\{\cdot\}$ in (11) is replaced by the imaginary part $\operatorname{Im}\{\cdot\}$.

The two-stage group detection can be structured as the group detection, then the element detection to find $A_{j / e}$ from the group symbol $A_{j}$. First, the group detection to search for $A_{j}, j=$ $0, \ldots, 63$ is performed by choosing

$$
\max _{\substack{\left(e_{l}=0,1,2,3\right) \\ l=0,1,2}}\left\{\rho_{G}\left(n ;\left\{e_{l}\right\}\right)=\frac{1}{\mathrm{MG}_{i}} \sum_{l=0}^{3}\left|\alpha_{e_{l}} \cdot X_{l}(n) \cdot \mathbf{1}_{G_{i}}^{T}\right|\right\} .
$$

Thus, the group detection can be viewed as a kind of envelope detection to be applied to the $M=16$ parallel MC channels, which requires 64-ary (6-b) symbol detection. It turns out that all distinct elements $\left\{A_{j / e}\right\}$ of each group yield the same decision variable $\rho_{G}\left(n ;\left\{e_{l}\right\}\right)$.

Next, suppose that $A_{j^{\prime}}=\left[\alpha_{e_{0}}\left|\alpha_{e_{1}}\right| \alpha_{e_{2}} \mid \alpha_{e_{3}}\right]$ is selected as the group symbol in (12). Then, the element detection can easily be carried out by choosing

$$
\max _{(e=0, \ldots, 7)}\left\{\rho_{E}\left(n ; j^{\prime}, e\right)=\frac{1}{\mathrm{MG}_{i}}\left(A_{j^{\prime} / e} \cdot X(n) \cdot \mathbf{1}_{G_{i}}^{T}\right)\right\}
$$

where the matrix $X(n)\left(M \times G_{i}\right)$ is $X(n)=$ $\left[X_{0}^{T}(n)\left|X_{1}^{T}(n)\right| X_{2}^{T}(n) \mid X_{3}^{T}(n)\right]^{T}$. Here, the element detection requires only calculation of the decision variables $\left\{\rho_{E}\left(n ; j^{\prime}, e\right) \mid e=1,2,3\right\}$, since $\rho_{E}\left(n ; j^{\prime}, 0\right)=\rho_{G}\left(n ;\left\{e_{l}\right\}\right)$ and $\rho_{E}\left(n ; j^{\prime}, e\right)=-\rho_{E}\left(n ; j^{\prime}, e-4\right)$ for $e=4,5,6,7$.

Finally, a symbol-by-symbol detection for the MDMC signaling in (4) was performed by choosing [9]

$$
\max _{\substack{(e=0, \ldots, 7) \\(f=0, \ldots, G-1)}}\left\{\rho_{\langle m\rangle}(n ; e, f)=\frac{1}{\mathrm{JG}}\left(\alpha_{e} \cdot X_{\langle m\rangle}(n) \cdot \mathbf{h}_{f}^{T}\right)\right\}
$$

where $\mathbf{h}_{f}$ is an $G$-ary orthogonal sequence of size $G$ with elements \pm 1 and decision statistics are found in (10) only by replacing $P_{i}, T_{i}$, and $G_{i}$ with $P, T$, and $G$. 
In view of receiver complexity, the proposed hybrid MC/VSF scheme employs 64-ary (6-b) symbol detection in the group detection when $M=16$ parallel MC channels are assumed, while the following element detection is based on 8-ary (3-b) symbol detection. The MDMC scheme also requires 64-ary (6-b) symbol detection when $M=16, J=4$, and $G=8$ are assumed in (14). Therefore, the proposed hybrid scheme causes a modest increase in receiver complexity compared to that of the MDMC, but the element detection can in fact be simplified since only three decision variables need to be computed. It is to be noted that the uplink of IS-95 CDMA system also adopts 64-ary orthogonal modulation [12] with $N=128$, resulting in the same order of receiver complexity as the MDMC and MC/VSF schemes. Meanwhile, the VSF and MC schemes employ a simple bit-by-bit detection so that they have a certain advantage of easy implementation.

\section{ANALYSIS OF HYBRID MC/VSF}

The hybrid MC/VSF scheme with two-stage group detection is analyzed in view of the SER when the precoding of rate 9/16 is adopted in $M=16$ parallel MC channels for a constant envelope. Let us define

$$
\rho_{G}\left(n ; e_{l}\right)=\frac{1}{\mathrm{MG}_{i}}\left|\alpha_{e_{l}} \cdot X_{l}(n) \cdot \mathbf{1}_{G_{i}}^{T}\right|
$$

for $l=0,1,2,3$, where $\rho_{G}\left(n ;\left\{e_{l}\right\}\right)=\sum_{l=0}^{3} \rho_{G}\left(n ; e_{l}\right)$. Assume that $\pm \alpha_{e_{l}}$ was sent; then, the decision variable $\rho_{G}\left(n ; e_{l}\right)$ can be written as

$$
\rho_{G}\left(n ; e_{l}\right)=\left.\frac{1}{4}\left|\sum_{v=1}^{2 V}\right| \beta_{1, v}\right|^{2}+I_{l}\left(e_{l}\right) \mid
$$

where the composite interference $I_{l}\left(e_{l}\right)$ results from the otheruser interference and the $I / Q$-channel self-interference

$$
I_{l}\left(e_{l}\right)=\sum_{k=2}^{K} \operatorname{MAI}_{k}\left(e_{l}\right)+\sum_{v=1}^{2 V}\left[\operatorname{MAI}_{1, v}^{(I)}\left(e_{l}\right)+\operatorname{MAI}_{1, v}^{(Q)}\left(e_{l}\right)\right] \text {. }
$$

Following Appendix A in [9] with $h_{e, g}=1$, it can be shown that

$$
\begin{aligned}
\mathbf{E}\left\{I_{l}^{2}\left(e_{l}\right)\right\} \triangleq & \sigma_{I_{l}}^{2} \\
= & \frac{1}{2 N_{i}}\left(\sum_{v=1}^{2 V}\left|\beta_{1, v}\right|^{2}\right) \sigma_{\beta}^{2} \\
& \cdot\left[(K-1) V \varepsilon_{i}^{2}+M(V-1)\right] \\
& \cdot \mathbf{E}\left\{\bar{\eta}^{2}(\delta)\right\} \\
\operatorname{covar}\left[I_{l}\left(e_{l}\right), I_{l}\left(e_{l}^{\prime}\right)\right]= & \mathbf{E}\left\{I_{l}\left(e_{l}\right) I_{l}\left(e_{l}^{\prime}\right)\right\} \\
= & \frac{1}{4}\left[\alpha_{e_{l}} \cdot\left(\alpha_{e_{l}^{\prime}}\right)^{T}\right] \sigma_{I_{l}}^{2}
\end{aligned}
$$

for identically distributed channel statistics $\mathbf{E}\left\{\left|\beta_{k, v}\right|^{2}\right\}=\sigma_{\beta}^{2}$ and $\varepsilon_{i}^{2} \triangleq \Gamma_{k} / P_{i}(k \geq 2)$. Here, the partial chip-pulse correlation is defined by $\bar{\eta}(\delta)=1 / T_{c} \int_{0}^{\delta} p(t) p\left(t-\delta+T_{c}\right) d t, \delta$ uniformly distributed over $\left[0, T_{c}\right)$. Note that if $\alpha_{e_{l}} \neq \pm \alpha_{e_{l}^{\prime}}$, then $\operatorname{covar}\left[I_{l}\left(e_{l}\right), I_{l}\left(e_{l}^{\prime}\right)\right]=0$, namely, uncorrelated. In addition, by the orthogonality among $M$ parallel MC channels, it becomes $\operatorname{covar}\left[I_{l}\left(e_{l}\right), I_{l^{\prime}}\left(e_{l^{\prime}}\right)\right]=0$, uncorrelated if $l \neq l^{\prime}$.
For the two-stage group detection, the probability of symbol error incurred by the group detection in (12) is defined by

$$
\begin{aligned}
P_{G}(\epsilon)=\operatorname{Pr}\left[\bigcup_{1 \leq f \leq 3} \bigcup_{\substack{\left(e_{l_{0}}, \ldots, e_{l_{f}}\right) \\
\times\left(e_{l_{0}}^{\prime}, \ldots, e_{l_{f}}^{\prime}\right)}} \sum_{i=0}^{f} \rho_{G}\left(n ; e_{l_{i}}\right)\right. \\
\left.\quad<\sum_{i=0}^{f} \rho_{G}\left(n ; e_{l_{i}}^{\prime}\right) \mid \pm \alpha_{e_{l_{i}}}, \quad 0 \leq l_{i} \leq 3\right]
\end{aligned}
$$

where $\alpha_{e_{l_{i}}} \neq \pm \alpha_{e_{l_{i}}^{\prime}}$ for $0 \leq l_{i} \leq 3$ and the decision variable $\rho_{G}\left(n ; e_{l_{i}}^{\prime}\right)$ simply reduces to

$$
\rho_{G}\left(n ; e_{l_{i}}^{\prime}\right)=\frac{1}{4}\left|I_{l_{i}}\left(e_{l_{i}}^{\prime}\right)\right|
$$

while $\rho_{G}\left(n ; e_{l_{i}}\right)$ is given by (16) with $l$ replaced by $l_{i}$. Let us define the set

$$
\begin{aligned}
\mathcal{L}_{f} \triangleq\left\{\left(e_{l_{0}}, \ldots, e_{l_{f}}\right) \times\left(e_{l_{0}}^{\prime}, \ldots, e_{l_{f}}^{\prime}\right) \mid \alpha_{e_{l_{i}}} \neq \pm \alpha_{e_{l_{i}}^{\prime}},\right. \\
\left.0 \leq l_{i} \leq 3\right\}
\end{aligned}
$$

for $f=1,2,3$ ( $\times$ denotes the cartesian product) and it is shown in Appendix A that $\left|\mathcal{L}_{1}\right|=18, \quad\left|\mathcal{L}_{2}\right|=24, \quad\left|\mathcal{L}_{3}\right|=21$ where $\sum_{f=1}^{3}\left|\mathcal{L}_{f}\right|=\left|\left\{A_{j}\right\}\right|-1=63$ except $A_{j^{\prime}}$, desired group symbol. By the precoding rule from (9), if there is a mismatch as $\alpha_{e_{l_{i}}} \neq \pm \alpha_{e_{l_{i}}^{\prime}}$ for some $l_{i}$, there should be another mismatch for $l_{i^{\prime}} \neq l_{i}$, which means $f \geq 1$ in (20).

Since $\left|\mathcal{L}_{f}\right| \gg 1$ for $f=1,2,3$, there exists a lot of dependency due to $\rho_{G}\left(n ; e_{l_{i}}\right)$ for any $e_{l_{i}}$ belonging to two elements in the same set $\mathcal{L}_{f}$ or different sets $\mathcal{L}_{f}$ and $\mathcal{L}_{f^{\prime}}$ for $f \neq f^{\prime}$. For this reason, it is highly expected that the pairwise error probability for two disjoint and mutually uncorrelated elements, namely, $\left(e_{0}, e_{1}\right)$ and $\left(e_{2}, e_{3}\right)$ in $\mathcal{L}_{1}(f=1)$ as a lower bound, can be a good approximation to $P_{G}(\epsilon)^{2}$

$$
\begin{aligned}
P_{G}(\epsilon) \approx & 2 \operatorname{Pr}\left[\rho_{G}\left(n ; e_{0}\right)+\rho_{G}\left(n ; e_{1}\right)\right. \\
& \left.<\rho_{G}\left(n ; e_{0}^{\prime}\right)+\rho_{G}\left(n ; e_{1}^{\prime}\right) \mid \pm \alpha_{e_{l}}, \quad l=0,1\right]
\end{aligned}
$$

which is validated by the simulation. Substituting (16) and (21) into (22) gives the bound

$$
\begin{aligned}
P_{G}(\epsilon) \approx 2 \operatorname{Pr} & {\left[\left|1+\bar{I}_{0}\left(e_{0}\right)\right|+\left|1+\bar{I}_{1}\left(e_{1}\right)\right|\right.} \\
& \left.<\left|\bar{I}_{0}\left(e_{0}^{\prime}\right)\right|+\left|\bar{I}_{1}\left(e_{1}^{\prime}\right)\right| \pm \alpha_{e_{l}}, \quad l=0,1\right]
\end{aligned}
$$

where $\bar{I}_{l}\left(e_{l}\right)=I_{l}\left(e_{l}\right) /\left(\sum_{v=1}^{2 V}\left|\beta_{1, v}\right|^{2}\right)$ with second moment $\mathbf{E}\left\{\bar{I}_{l}^{2}\left(e_{l}\right)\right\}=\gamma^{-1}$ and per-symbol signal-to-interference ratio (SIR) $\gamma$ is evaluated as

$$
\gamma=\left(\frac{\sum_{v=1}^{2 V}\left|\beta_{1, v}\right|^{2}}{\sigma_{\beta}^{2}}\right)\left[\frac{2 N_{i}}{\left[(K-1) V \varepsilon_{i}^{2}+M(V-1)\right] \mathbf{E}\left\{\bar{\eta}^{2}(\delta)\right\}}\right] .
$$

\footnotetext{
${ }^{2}$ All other elements in $\mathcal{L}_{f}, f=1,2,3$, are partially correlated with the two elements in terms of $\rho_{G}\left(n ; e_{l_{i}}\right)$.
} 
It is assumed that the composite interferences $\left\{\bar{I}_{l}\left(e_{l}\right)\right\}$ are dominant in uplink high-rate DS-CDMA transmission, which are modeled as Gaussian because of the multipath-induced interferences. Conditioned on $\gamma$ in (24), the probability of symbol error can be derived in Appendix B as

$$
\begin{aligned}
P_{G}(\epsilon \mid \gamma) \approx & 16 \pi^{-2} \int_{0}^{\infty} \int_{0}^{\infty} \int_{\pi / 4}^{\pi / 2} \gamma \exp \left[-\frac{\gamma(x+y)^{2}}{4 \sin ^{2} \theta}\right] d \theta \\
& \cdot \exp \left[-\frac{\gamma\left(x^{2}+y^{2}+2\right)}{2}\right] \cosh (\gamma x) \\
& \cdot \cosh (\gamma y) d x d y .
\end{aligned}
$$

Let define $\bar{\gamma}=\gamma / \Omega$, where

$$
\Omega=\left[\frac{2 N_{i}}{\left[(K-1) V \varepsilon_{i}^{2}+M(V-1)\right] \mathbf{E}\left\{\bar{\eta}^{2}(\delta)\right\}}\right] .
$$

Then, the probability density function (pdf) of $\bar{\gamma}$ is derived as [13]

$$
f_{\bar{\gamma}}(s)=\frac{s^{2 V-1}}{(2 V-1) !} e^{-s}, \quad s \geq 0
$$

for identically distributed channel statistics.

Now, if an average of (25) is taken with respect to $\bar{\gamma}$, it can be further evaluated in Appendix $\mathrm{C}$ as

$$
\begin{aligned}
P_{G}(\epsilon) \approx 32 \pi^{-2} & \cdot \int_{\pi / 4}^{\pi / 2} \int_{0}^{\pi / 2}\left\{\frac{\Psi(\theta, \phi)}{(1+\Omega)^{2 V}}+\frac{(4 V-1) ! !}{(2 V-1) !(2 \Omega)^{2 V}}\right. \\
& \cdot\left[\Xi_{+}(\theta, \phi ; \Omega ; 2 V)\right. \\
& \left.\left.+\Xi_{-}(\theta, \phi ; \Omega ; 2 V)\right]\right\} d \theta d \phi
\end{aligned}
$$

where $(2 n-1) ! !=1 \cdot 3 \cdot 5 \cdots(2 n-1)$ and

$$
\begin{aligned}
& \Xi_{ \pm}(\theta, \phi ; \Omega ; n)= \sqrt{\Phi_{ \pm}(\theta, \phi)} \Psi(\theta, \phi) \\
& \cdot \int_{0}^{\pi / 2}\left\{\left[1+\Omega^{-1}-\Phi_{ \pm}(\theta, \phi)\right]^{-n-1 / 2}\right. \\
&-\left[1+\Omega^{-1}-\Phi_{ \pm}(\theta, \phi)\right. \\
&\left.\left.\cdot\left(1-\sin ^{-2} \psi\right)\right]^{-n-1 / 2}\right\} d \psi
\end{aligned}
$$

for $\Psi(\theta, \phi) \triangleq \sin ^{2} \theta /\left(1+2 \sin ^{2} \theta+\sin 2 \phi\right)$ and $\Phi_{ \pm}(\theta, \phi) \triangleq$ $(\cos \phi \pm \sin \phi)^{2} \Psi(\theta, \phi)$.

Next, nonidentically distributed channel statistics are considered to evaluate $P_{G}(\epsilon)$ in (23). It is assumed that the path gains with same delay are the same for two antennas with $\mathbf{E}\left\{\left|\beta_{k, v+q V}\right|^{2}\right\}=\sigma_{\beta}^{2}(v)[14]$. Then, it becomes $\gamma=\sum_{v=1}^{2 V} \gamma_{v}$, where

$$
\mathbf{E}\left\{\gamma_{v+q V}\right\} \triangleq \Omega_{v}=\left(\frac{\sigma_{\beta}^{2}(v)}{\frac{1}{V} \sum_{l=1}^{V} \sigma_{\beta}^{2}(l)}\right) \Omega
$$

for $v=1, \ldots, V ; q=0,1$. Applying the characteristic function method in [15], the pdf of $\gamma$ can be derived as

$$
\begin{aligned}
f_{\gamma}(s)=\sum_{v=1}^{V} \frac{\pi_{v}^{2}}{\Omega_{v}^{2}} s \exp \left(-\frac{s}{\Omega_{v}}\right)+\sum_{v=1}^{V} \sum_{\substack{v \prime=1 \\
v \neq v}}^{V} \frac{\pi_{v} \pi_{v^{\prime}}}{\Omega_{v}-\Omega_{v^{\prime}}} \\
\cdot\left[\exp \left(-\frac{s}{\Omega_{v}}\right)-\exp \left(-\frac{s}{\Omega_{v^{\prime}}}\right)\right]
\end{aligned}
$$

where $\pi_{v}=\prod_{\substack{v^{\prime}=1 \\ v^{\prime} \neq v}}^{V} \Omega_{v} /\left(\Omega_{v}-\Omega_{v^{\prime}}\right)$.

By taking the average of (25) with $f_{\gamma}(s)$, it follows that (see (31) at the bottom of the page).

Finally, the element detection in (13) gives rise to a symbol error even if the group detection is correctly made, which is defined by

$$
P_{E}(\epsilon \mid \zeta)=\operatorname{Pr}\left[\bigcup_{\substack{0 \leq e^{\prime} \leq 7 \\ e^{\prime} \neq e}} \rho_{E}\left(n ; j^{\prime}, e\right)<\rho_{E}\left(n ; j^{\prime}, e^{\prime}\right) \mid A_{j^{\prime} / e}, \zeta\right]
$$

for the event $\zeta$ as a correct group detection, i.e.,

$$
\left.>\sum_{i=0}^{f} \rho_{G}\left(n ; e_{l_{i}}^{\prime}\right) \mid \pm \alpha_{e_{l_{i}}}, 0 \leq l_{i} \leq 3\right\} .
$$

It is noted that

$$
P_{E}(\epsilon \mid \zeta) \leq \frac{P_{E}(\epsilon)}{1-P_{G}(\epsilon)} \leq 2 P_{E}(\epsilon)
$$

because of $\operatorname{Pr}[\zeta]=1-P_{G}(\epsilon) \geq 1 / 2$.

$$
\begin{aligned}
P_{G}(\epsilon) \approx 32 \pi^{-2} \int_{\pi / 4}^{\pi / 2} \int_{0}^{\pi / 2}\left\{\sum_{v=1}^{V}\right. & \frac{\pi_{v}^{2} \Psi(\theta, \phi)}{\left(1+\Omega_{v}\right)^{2}}+\sum_{v=1}^{V} \sum_{\substack{v^{\prime}=1 \\
v^{\prime} \neq v}}^{V} \frac{\pi_{v} \pi_{v^{\prime}} \Psi(\theta, \phi)}{\left(1+\Omega_{v}\right)\left(1+\Omega_{v^{\prime}}\right)} \\
& +\sum_{v=1}^{V} \frac{3 \pi_{v}^{2}}{4 \Omega_{v}^{2}}\left[\Xi_{+}\left(\theta, \phi ; \Omega_{v} ; 2\right)+\Xi_{-}\left(\theta, \phi ; \Omega_{v} ; 2\right)\right] \\
& +\sum_{v=1}^{V} \sum_{\substack{v^{\prime}=1 \\
v^{\prime} \neq v}}^{V} \frac{\pi_{v} \pi_{v^{\prime}}}{2\left(\Omega_{v}-\Omega_{v^{\prime}}\right)}\left[\Xi_{+}\left(\theta, \phi ; \Omega_{v} ; 1\right)-\Xi_{+}\left(\theta, \phi ; \Omega_{v^{\prime}} ; 1\right)\right. \\
& \left.\left.+\Xi_{-}\left(\theta, \phi ; \Omega_{v} ; 1\right)-\Xi_{-}\left(\theta, \phi ; \Omega_{v^{\prime}} ; 1\right)\right]\right\} d \theta d \phi .
\end{aligned}
$$




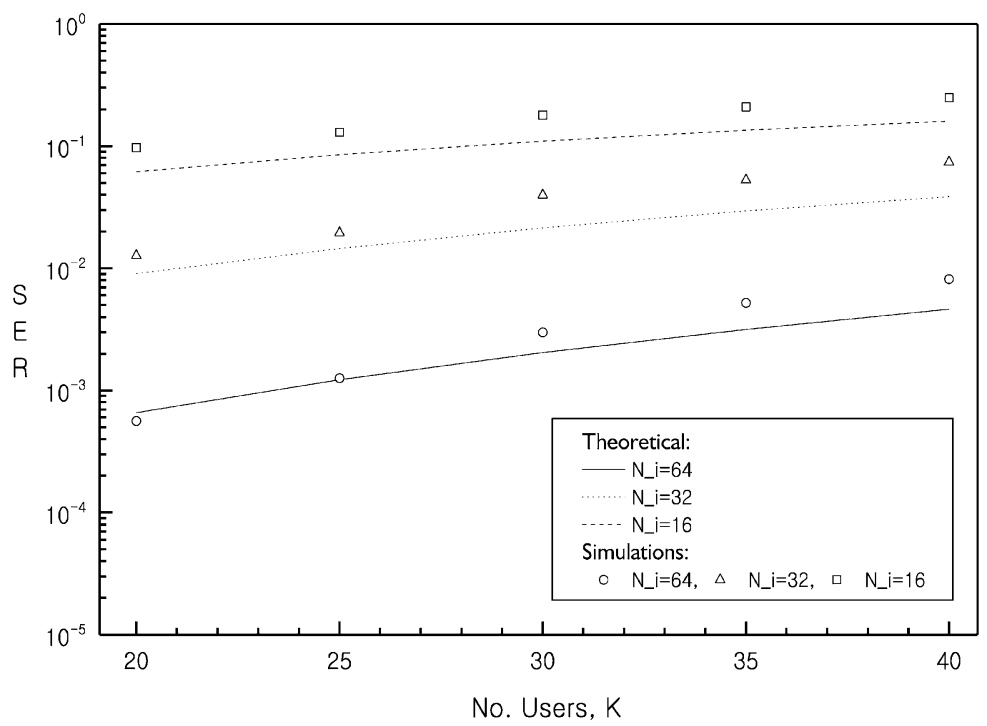

Fig. 1. SER versus $K$ for the hybrid MC/VSF under equal path gain.

Similarly, as in (22) and (23), $P_{E}(\epsilon)$ is derived by the pairwise error probability, i.e.,

$$
\begin{aligned}
P_{E}(\epsilon) \approx 2 \operatorname{Pr}[1+ & \bar{I}_{0}\left(e_{0}\right)+1+\bar{I}_{1}\left(e_{1}\right) \\
& \left.<-1-\bar{I}_{0}\left(e_{0}\right)-1-\bar{I}_{1}\left(e_{1}\right) \mid A_{j^{\prime} / 0}\right]
\end{aligned}
$$

where $\alpha_{e_{l_{i}}^{\prime}}=-\alpha_{e_{l_{i}}}$ has been assumed for $0 \leq l_{i} \leq 3$ with $\left|\mathcal{L}_{1}\right|=6$ for two mismatches. Conditioned on $\gamma$, it can be evaluated as

$$
P_{E}(\epsilon \mid \gamma) \approx 2 Q(\sqrt{2 \gamma})
$$

which yields $P_{E}(\epsilon \mid \gamma, \zeta) \approx 4 Q(\sqrt{2 \gamma})$ from (33). It turns out that

$$
P_{G}(\epsilon \mid \gamma) \gg P_{E}(\epsilon \mid \gamma, \zeta)
$$

for typical values of $\gamma$ and, hence, the SER performance here is analyzed based on the group detection only.

\section{RESULTS}

To begin , key system parameters for performance evaluation are assumed as follows: number of $\mathrm{MC}$ channels $M=16$, SF $N=128$, number of users $K=20-40$, and number of paths $V=3$, where unequal path gains are given by $\sigma_{\beta}^{2}(1)=0.6$, $\sigma_{\beta}^{2}(2)=0.3$, and $\sigma_{\beta}^{2}(3)=0.1$ and the precoding of rate $9 / 16$ is adopted. Also, the power ratio $\varepsilon_{i}^{2} \triangleq \Gamma_{k} / P_{i}, k \geq 2$ is set to the value $\varepsilon_{i}^{2}=8 / 9$ with $M=16$ since $\Gamma_{k}=M P_{i} / R$ at the rate $R=18 \mathrm{~b}$ per $2 N_{i}$ chips by the hybrid MC/VSF, given $1 \mathrm{~b}$ per $N_{k}=2 N_{i}$ chips for a low-rate user in (5). Here, the data rates are varied depending on $N_{i}=64,32,16$ with $N_{i}=N / 2^{i}$, $i=1,2,3$, which result in the rates $18,36,72$ b per $N=128$ chips, respectively.

To fairly compare the hybrid MC/VSF scheme with MDMC, the precoding rule from (9) is exploited in both schemes. In fact, the group detection of the hybrid scheme was realized in connection with the precoding to minimize receiver complexity where the MRC with $2 V$ paths was employed in (11). Likewise, the MDMC scheme uses the error detection by precoding, which gives adaptive selection combining (SC)/MRC receiver as follows. First, the SC combines the strongest (highest SIR) $V=3$ paths among $2 V=6$ available ones for initial data detection. If there is an error detected, detection is repeated using the MRC with $2 V=6$ paths. It is seen by the simulation that some detection gain can be achieved using adaptive SC/MRC rather than MRC in case of the MDMC below.

First, theoretical results on the SER are presented for the group detection of the hybrid MC/VSF scheme in Figs. 1 and 2 for varying $N_{i}=64,32,16$ where equal and unequal path gains are assumed, respectively. Simulation results are also provided to see the performance behavior of the approximate bound on $P_{G}(\epsilon)$, as given in (22). The lower bound is not tight, but the relative behavior versus $N_{i}$ fits the simulation results well. It implies that the approximate bound is meaningful to find the degradation in SER as the SF $N_{i}$ is reduced to increase the data rate. Also, this observation is equally valid when either identically or nonidentically distributed channel statistics are considered. With $M=16$ parallel MC channels, the hybrid scheme can coexist with $K=40$ low-rate users at the high rate of $18 \mathrm{~b}$ per $N=128$ chips subject to the (uncoded) SER below $10^{-2}$, even for the unequal path gains. For example, if the low-rate carrying voice traffic is $8 \mathrm{~kb} / \mathrm{s}$, then the high rate of $144 \mathrm{~kb} / \mathrm{s}$ can be supported for data traffic with high (coded) QoS.

Next, the hybrid MC/VSF scheme is compared with MDMC, $\mathrm{MC}$, and VSF subject to the same data rate, i.e., $18 \mathrm{~b}$ per $N=$ 128 chips except VSF, in terms of the simulated BER with fixed $N_{i}=64$. Here, the VSF scheme conveys 16 b per $N=128$ chips to have an effective SF per bit that is an integer, namely, $8=128 / 16$ (chips/bit). To minimize envelope variations for the MC scheme, we adopt the precoder of rate 18/32 that is realized by two precoders of rate $9 / 16$ in parallel. In this case, the MC scheme has the peak-to-average ratio of two while the hybrid MC/VSF, MDMC, and VSF schemes have the peak-to-average ratio of one, because the constant envelope is maintained. Then, the BER would be deteriorated if nonlinear distortions are considered for the MC scheme, which are not considered here.

Fig. 3 shows the corresponding BER curves versus $K$ under equal path gain, which verifies the detection gain by the 


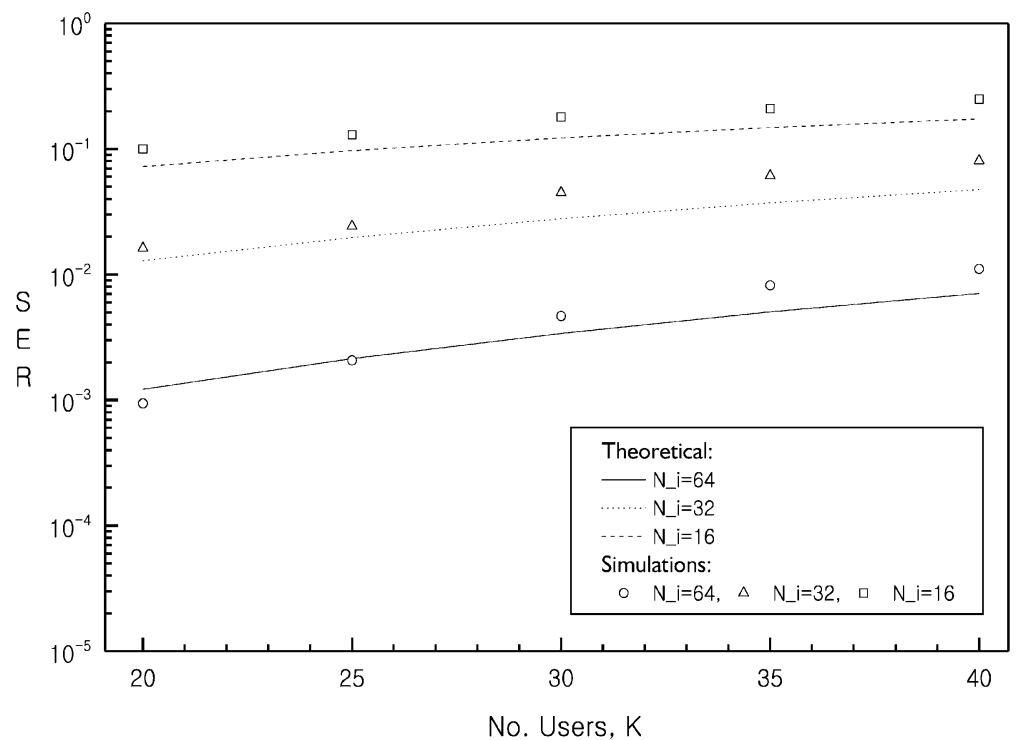

Fig. 2. SER versus $K$ for the hybrid MC/VSF under unequal path gains.

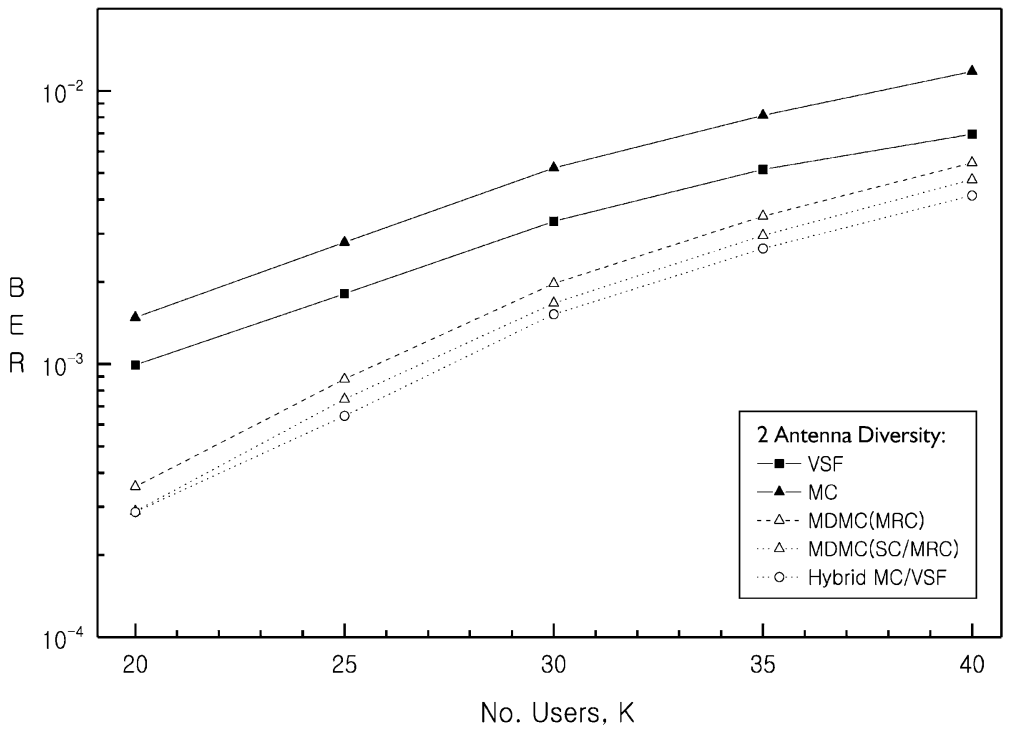

Fig. 3. BER versus $K$ for performance comparison under equal path gain.

two-stage group detection. As the number of users $K$ decreases, the MDMC scheme with adaptive SC/MRC is comparable to the hybrid scheme or even performs better for $K<20$. However, the hybrid scheme gives slightly better BER performance for the medium range of $K>20$ due to the $M$-symbol detection while $J$-symbol detection was used for the MDMC scheme. For comparison, the MDMC scheme with MRC is also included in the BER curves, which identify a loss in diversity gain. Besides, it is observed that conventional MC and VSF schemes are far inferior to the MDMC and hybrid $\mathrm{MC} / \mathrm{VSF}$ schemes, but the performance gain is reduced for large $K$, compared to the VSF scheme. This is because the high-order modulation is more sensitive to the low-rate user interference compared to the binary signaling of the VSF scheme. Hence, it is necessary to employ some techniques to mitigate the other-user interference (for instance, multiuser receivers in [16]) by which the detection gain can be fully assured for the $M$-symbol group detection.

In the case of unequal path gains, the BER curves are shown in Fig. 4, in which the same performance behavior is observed as in Fig. 3. It is important to note that the combined effects of worse fading and large multiple-access interference (MAI) are detrimental, especially to the MDMC. But the hybrid MC/VSF scheme provides a certain gain in BER even though the gain is much reduced here for large $K$. Also, it is noted that the BER curve would be a bit deteriorated for the VSF scheme if the data rate is kept the same.

Finally, the BER performance comparison as a function of $N_{i}$ is carried out for the hybrid MC/VSF and VSF schemes. With an integer-effective SF per bit, the data rates of 16, 32, and $64 \mathrm{~b}$ per $N=2^{i} N_{i}=128$ chips are possible for the VSF scheme when $N_{i}=64,32,16$ for $i=1,2,3$, respectively. Thus, the hybrid scheme sends additional 2, 4, and 8 b for the corresponding $N_{i}$ in the $M=16$ parallel MC channels. It is found in Figs. 5 and 6 that additional $12.5 \%$ gain in data rate can be achieved for the hybrid scheme subject to $N_{i} \geq 32$, while the gain is not supported with lower $N_{i}=16$. It can be interpreted that the processing gain is not enough to realize the detection gain by the $M$-symbol group detection in an interference-limited uplink channel with multipath fading, especially when $N_{i} \rightarrow M$. 


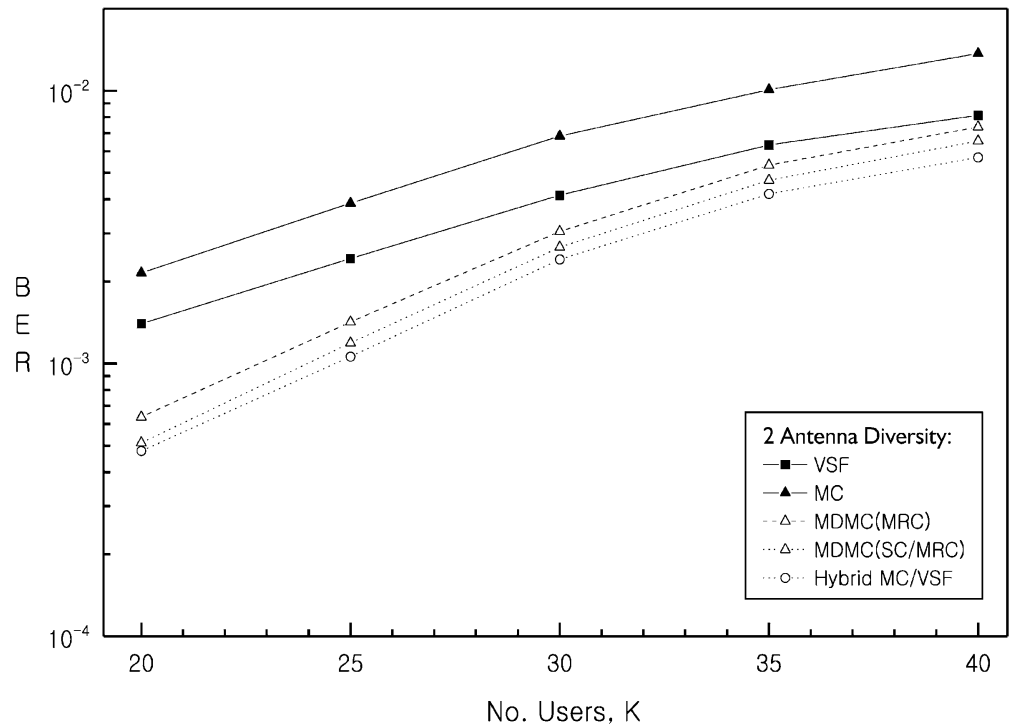

Fig. 4. BER versus $K$ for performance comparison under unequal path gains.

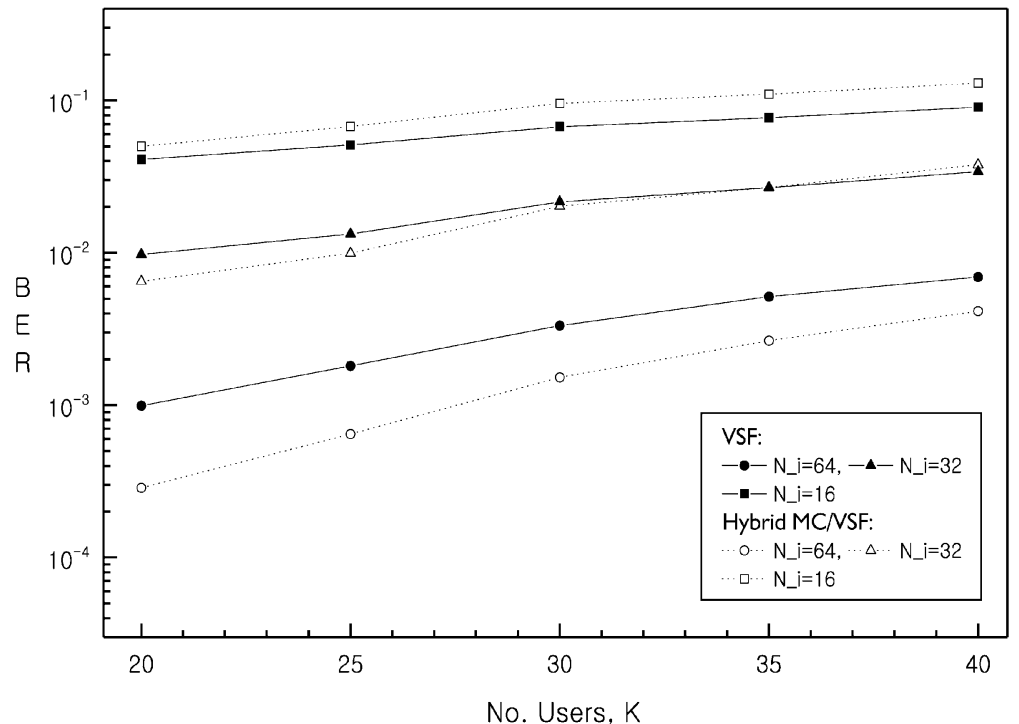

Fig. 5. BER versus $K$ for performance comparison as a function of $N_{i}$ under equal path gain.

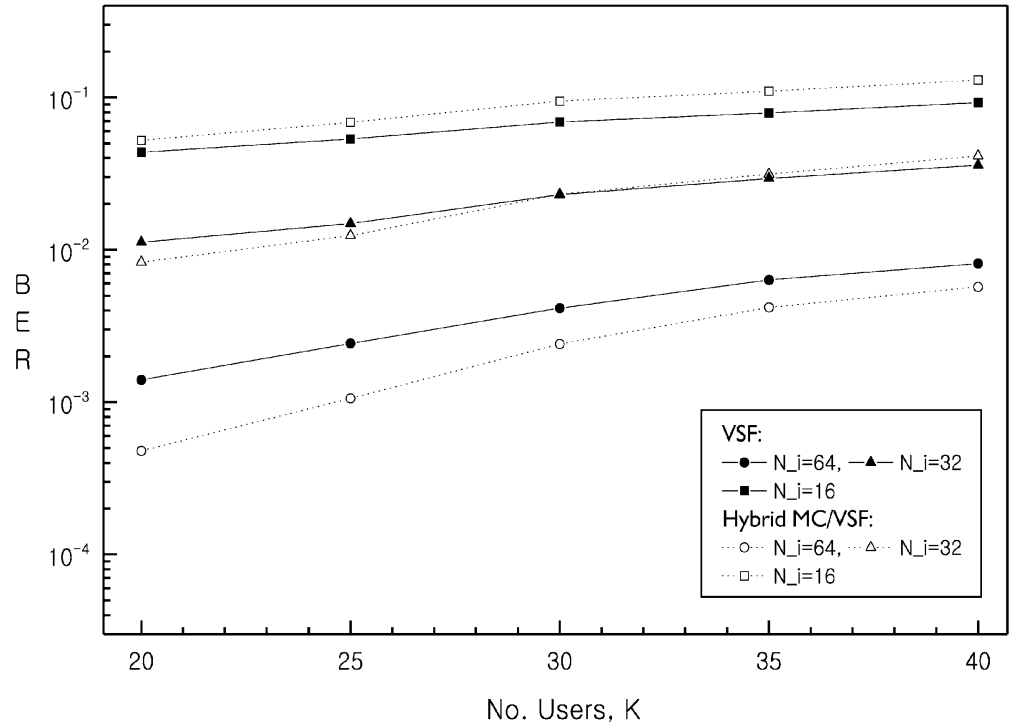

Fig. 6. BER versus $K$ for performance comparison as a function of $N_{i}$ under unequal path gains. 


\section{CONCLUSION}

For reliable high-rate uplink transmission, a hybrid MC/VSF scheme has been proposed to fulfill a compromise between the minimum SF and the maximum number of MC channels. This realization is based on the characteristics of uplink channel, namely power limitation and nonlinear distortion, which require a certain SF via MC transmission. For this reason, precoding to minimize envelope variations as a result of MC option is prerequisite for uplink power-efficient channel. This underlying fact has been motivated to realize the two-stage $M$-symbol group detection to recover some signal energy loss by precoding.

First, it was shown that the hybrid scheme assures a certain detection gain by the use of the $M$-symbol group detection. Second, the detection gain can fully be achieved by mitigating the combined effects of MAI and multipath fading. Especially, some reduction in the MAI using the multiuser receiver is suggested in connection with the group detection. Also, multiple receive antenna diversity may be effective to make the group detection attractive to the (base) receiver. Third, it was found that the hybrid scheme can provide additional $12.5 \%$ gain in data rate compared to the VSF, with SF exceeding the number of MC channels.

\section{APPENDIX A}

\section{CARdinality OF THE SET $\mathcal{L}_{f}$}

First, to find $\mathcal{L}_{1}$, any two positions with mismatch are selected in $\left(\begin{array}{l}4 \\ 2\end{array}\right)$ ways, where $\left(\begin{array}{l}n \\ k\end{array}\right) \triangleq n ! /(n-k) ! / k !$. Suppose that $A_{j^{\prime}}=\left[\alpha_{e_{0}}\left|\alpha_{e_{1}}\right| \alpha_{e_{2}} \mid \alpha_{e_{3}}\right]$ was chosen as the group symbol in (12). Then, there are three different symbols $\alpha_{e_{l}^{\prime}} \neq \alpha_{e_{l}}$ for $0 \leq$ $e_{l} \leq 3(l \neq 3)$ as the group symbol in one position, but the symbol in the other position is fixed by the precoding rule from (9). This implies that $\left|\mathcal{L}_{1}\right|=3\left(\begin{array}{l}4 \\ 2\end{array}\right)=18$.

Next, any three positions with mismatch are selected to find $\mathcal{L}_{2}$ in $\left(\begin{array}{l}4 \\ 3\end{array}\right)$ ways and then six different combinations can be placed into the three positions. For instance, if $e_{0}=e_{1}=e_{2}=$ 0 with $e_{3}=7$ and $l=1,2,3$ are the three positions with mismatch, then any one of $e_{1}^{\prime}=1,2,3$ can be chosen but $e_{2}^{\prime} \neq e_{1}^{\prime}$ and otherwise, i.e., if $e_{2}^{\prime}=e_{1}^{\prime}$, then $e_{3}^{\prime}=e_{3}=7$, contradiction! So there are two cases for each $e_{1}^{\prime}=1,2,3$, resulting in six combinations. This produces $\left|\mathcal{L}_{2}\right|=6\left(\begin{array}{l}4 \\ 3\end{array}\right)=24$.

Finally, using the fact that $\left|\left\{A_{j}, j \neq j^{\prime}\right\}\right|=\sum_{f=1}^{3}\left|\mathcal{L}_{f}\right|=63$ yields $\left|\mathcal{L}_{3}\right|=63-(18+24)=21$.

\section{APPENDIX B}

DERIVATION OF $P_{G}(\epsilon \mid \gamma)$ IN (25)

From (20) and by definition on $\mathcal{L}_{f}$, the union bound can be expressed by

$$
\begin{aligned}
P_{G}(\epsilon) \leq \sum_{f=1}^{3}\left|\mathcal{L}_{f}\right| & \operatorname{Pr}\left[\sum_{l=0}^{f} \rho_{G}\left(n ; e_{l}\right)\right. \\
& \left.<\sum_{l=0}^{f} \rho_{G}\left(n ; e_{l}^{\prime}\right) \mid \pm \alpha_{e_{l}}, \quad 0 \leq l \leq 3\right] .
\end{aligned}
$$

Since most probable errors result from the events defined on $\mathcal{L}_{1}$ and there is a lot of dependency among elements even in $\mathcal{L}_{1}$, $P_{G}(\epsilon)$ is well approximated by the pairwise error probability for the two disjoint and mutually uncorrelated elements in $\mathcal{L}_{1}$, as given in (22). It can be formulated as

$$
P_{G}(\epsilon) \approx 2 \operatorname{Pr}\left[\xi_{0}+\xi_{1}<\left|\lambda_{0}\right|+\left|\lambda_{1}\right| \mid \pm \alpha_{e_{l}}, \quad l=0,1\right]_{38}
$$

by (23) along with $\xi_{l} \triangleq\left|1+\bar{I}_{l}\left(e_{l}\right)\right|$ and $\lambda_{l} \triangleq \bar{I}_{l}\left(e_{l}^{\prime}\right)$.

It is simply evaluated as

$$
\begin{aligned}
\frac{P_{G}(\epsilon)}{2} \approx 1-\mathbf{E}\left\{\operatorname { P r } \left[\left|\lambda_{0}\right|+\left|\lambda_{1}\right|<x+y\right.\right. \\
\\
\left.\left.\quad \mid \xi_{0}=x, \xi_{1}=y, \pm \alpha_{e_{l}}, l=0,1\right]\right\} .
\end{aligned}
$$

Conditioned on $\gamma$ and through a rotation of the axes $\lambda_{0}$ and $\lambda_{1}$ by $\pi / 4$, it is further evaluated as

$$
\begin{aligned}
\operatorname{Pr}\left[\left|\lambda_{0}\right|+\left|\lambda_{1}\right|<x+y \mid \xi_{0}\right. & \left.=x, \xi_{1}=y, \pm \alpha_{e_{l}}, l=0,1\right] \\
& =\left[1-2 Q\left(\frac{x+y}{\sqrt{2 \gamma^{-1}}}\right)\right]^{2}
\end{aligned}
$$

where $f_{\lambda_{l}}(u)=1 / \sqrt{2 \pi \gamma^{-1}} \exp \left[-u^{2} /\left(2 \gamma^{-1}\right)\right]$ and $Q(z)=$ $\int_{z}^{\infty} f_{\lambda_{l}}(u) d u$ with $\gamma=1$.

By taking the expectation in (39) with respect to $\xi_{l}(l=0,1)$, it follows that

$$
\begin{aligned}
P_{G}(\epsilon \mid \gamma) \approx & 8 \int_{0}^{\infty} \int_{0}^{\infty}\left[f_{\xi_{0}}(x)+f_{\xi_{0}}(-x)\right] \\
& \cdot\left[f_{\xi_{1}}(y)+f_{\xi_{1}}(-y)\right] \\
& \cdot\left[Q\left(\frac{x+y}{\sqrt{2 \gamma^{-1}}}\right)-Q^{2}\left(\frac{x+y}{\sqrt{2 \gamma^{-1}}}\right)\right] d x d y
\end{aligned}
$$

where $f_{\xi_{l}}(x)=1 / \sqrt{2 \pi \gamma^{-1}} \exp \left[-(x-1)^{2} /\left(2 \gamma^{-1}\right)\right]$. Then, applying the definite integral forms such as [17]

$$
\begin{aligned}
Q(z) & =\frac{1}{\pi} \int_{0}^{\pi / 2} \exp \left(-\frac{z^{2}}{2 \sin ^{2} \theta}\right) d \theta \\
Q^{2}(z) & =\frac{1}{\pi} \int_{0}^{\pi / 4} \exp \left(-\frac{z^{2}}{2 \sin ^{2} \theta}\right) d \theta
\end{aligned}
$$

$P_{G}(\epsilon \mid \gamma)$ can be evaluated as given in (25).

\section{APPENDIX C}

EVAluAtion of $P_{G}(\epsilon)$ IN (28)

By the change of variables $(x=r \cos \phi, y=r \sin \phi)$ in (25), it can be rewritten as

$$
\begin{aligned}
& P_{G}(\epsilon \mid \gamma) \approx 16 \pi^{-2} \int_{\pi / 4}^{\pi / 2} \int_{0}^{\pi / 2} \gamma e^{-\gamma} \\
& \cdot\left\{\int_{0}^{\infty} r \exp \left[-\frac{r^{2} \gamma\left(1+2 \sin ^{2} \theta+\sin 2 \phi\right)}{4 \sin ^{2} \theta}\right]\right. \\
&\cdot \cosh (r \gamma \cos \phi) \cosh (r \gamma \sin \phi) d r\} d \theta d \phi
\end{aligned}
$$




$$
\begin{aligned}
& P_{G}(\epsilon \mid \gamma) \approx 16 \pi^{-2} \int_{\pi / 4}^{\pi / 2} \int_{0}^{\pi / 2} e^{-\gamma} \\
& \cdot\{(2+\sqrt{\pi \gamma}) \Psi(\theta, \phi) \\
& \cdot\left[\sqrt{\Phi_{+}(\theta, \phi)} \exp \left(\gamma \Phi_{+}(\theta, \phi)\right)\right. \\
& \cdot \operatorname{erf}\left(\sqrt{\gamma \Phi_{+}(\theta, \phi)}\right)+\sqrt{\Phi_{-}(\theta, \phi)} \\
&\left.\left.\cdot \exp \left(\gamma \Phi_{-}(\theta, \phi)\right) \operatorname{erf}\left(\sqrt{\gamma \Phi_{-}(\theta, \phi)}\right)\right]\right\} \cdot d \theta d \phi
\end{aligned}
$$

Performing the integration on $r$ gives the finite integral form shown in (43) at the top of the page where $\operatorname{erf}(z)=1-$ $2 Q(\sqrt{2} z)$. Now, taking the average with respect to $\gamma=\bar{\gamma} \Omega$

$$
P_{G}(\epsilon)=\int_{0}^{\infty} P_{G}(\epsilon \mid \gamma=s \Omega) f_{\bar{\gamma}}(s) d s
$$

yields $P_{G}(\epsilon)$ in (28).

\section{REFERENCES}

[1] J. Chung et al., "High-speed wireless data access based on combining EDGE with wideband OFDM," IEEE Commun. Mag., vol. 37, pp. 92-98, Nov. 1999.

[2] F. Adachi, M. Sawahashi, and H. Suda, "Wideband DS-CDMA for nextgeneration mobile communications system," IEEE Commun. Mag., vol. 36, pp. 56-69, Sept. 1998.

[3] E. Dahlman et al., "UMTS/IMT-2000 based on wideband CDMA," IEEE Commun. Mag., vol. 36, pp. 70-80, Sept. 1998.

[4] I. Chih-Lin and R. D. Gitlin, "Multi-code CDMA wireless personal communications networks," in Proc. IEEE ICC '95, vol. 2/3, Seattle, WA, June 1995, pp. 1060-1064.

[5] I. Chih-Lin, G. P. Pollini, L. Ozarow, and R. D. Gitlin, "Performance of multi-code CDMA wireless personal communications networks," in Proc. IEEE VTC '95, Apr. 1995, pp. 907-911.

[6] T. Ottosson, "Precoding in multicode DS-CDMA systems," in Proc. IEEE ISIT '97, Ulm, Germany, June 1997, p. 351.

[7] T. Wada, T. Yamazato, M. Katayama, and A. Ogawa, "A constant amplitude coding for orthogonal multi-code CDMA systems," IEICE Trans. Fundam., vol. E80-A, pp. 2477-2484, Dec. 1997.

[8] T. Ottosson and T. Palenius, "The impact of using multicode transmission in the WCDMA system," in Proc. IEEE VTC '99, vol. 2, May 1999, pp. $1550-1554$

[9] D. I. Kim and V. K. Bhargava, "Performance of multidimensional multicode DS-CDMA using code diversity and error detection," IEEE Trans. Commun., vol. 49, pp. 875-887, May 2001.

[10] N. Guo and L. B. Milstein, "On rate-variable multidimensional DS/SSMA with sequence decoding," IEEE J. Select. Area. Commun., vol. 17, pp. 902-917, May 1999.

[11] J. Chen, J. Wang, and M. Sawahashi, "MCI cancellation for multicode wideband CDMA systems," IEEE J. Select. Area. Commun., vol. 20, pp. 450-462, Feb. 2002.
[12] A. J. Viterbi, CDMA: Principles of Spread Spectrum Communication. Reading, MA: Addison-Wesley, 1995.

[13] A. D. Whalen, Detection of Signals in Noise. New York: Academic, 1971.

[14] M. K. Simon and M.-S. Alouini, Digital Communication Over Fading Channels. New York: Wiley, 2000.

[15] J. G. Proakis, Digital Communications, 2nd ed. New York: McGrawHill, 1989.

[16] S. Verdú, Multiuser Detection. New York: Cambridge Univ. Press, 1988.

[17] M. K. Simon and D. Divsalar, "Some new twists to problems involving the Gaussian probability integral," IEEE Trans. Commun., vol. 46, pp. 200-210, Feb. 1998.

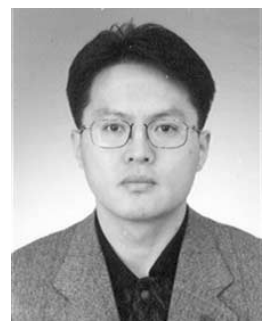

Dong In Kim (S'89-M'91-SM'02) received the B.S. and M.S. degrees in electronics engineering from Seoul National University, Seoul, Korea, in 1980 and 1984, respectively, and the M.S. and Ph.D. degrees in electrical engineering from University of Southern California (USC), Los Angeles, in 1987 and 1990, respectively.

From 1984 to 1985 , he was a Researcher with the Korea Telecom Research Center, Seoul, Korea. From 1986 to 1988, he was a Korean Government Graduate Fellow in the Department of Electrical Engineering, USC. From 1991 to 2002, he was with the University of Seoul, leading the Wireless Communications Research Group. He was a Visiting Professor with University of Victoria, Victoria, BC, Canada, from 1999 to 2000. Since 2002, he has been an Associate Professor with the School of Engineering Science, Simon Fraser University, Burnaby, BC, Canada. He has performed research in the areas of packet radio networks and spread-spectrum systems since 1988. His current research interests include spread-spectrum systems, cellular mobile communications, indoor wireless communications, and wireless multimedia networks.

Dr. Kim has served as an Editor for the IEEE JOURNAL ON SELECTED AREAS IN COMMUNICATIONS: WiRELESS COMMUNICATIONS SERIES and also as a Division Editor for the Journal of Communications and Networks. Currently, he serves as an Editor for the IEEE TRANSACTIONS ON COMMUNICATIONS and the IEEE TRANSACTIONS ON WIRELESS COMMUNICATIONS. 